\title{
Low-dose glucose infusion after achieving critical hypoglycemia during insulin tolerance testing: effects on time of hypoglycemia, neuroendocrine stress response and patient's discomfort in a pilot study
}

\author{
K Borm, M Slawik, F Beuschlein, L Seiler, F Flohr, A Berg ${ }^{1}$, A Koenig ${ }^{2}$ and M Reincke ${ }^{2}$ \\ Division of Endocrinology, Department of Medicine II and ${ }^{1}$ Department of Sports Medicine, University Hospital of Freiburg, Germany, ${ }^{2}$ Medical Clinic, \\ University Hospital Innenstadt, Ludwig Maximilians University, Ziemssenstrasse 1, 80336 Munich, Germany \\ (Correspondence should be addressed to M Reincke; Email: martin.reincke@med.uni-muenchen.de)
}

\begin{abstract}
Objective: The insulin tolerance test (ITT) is regarded as the gold standard for the evaluation of pituitary ACTH and growth hormone reserve. However, the intended critical hypoglycemia results in considerable discomfort and requires close surveillance during the test.

Design and methods: In a pilot study, we evaluated whether the ITT could be markedly simplified, made less hazardous and more convenient by routine i.v. low-dose glucose administration after hypoglycemia has been achieved. Sixteen healthy subjects (three females, 13 males) were tested twice in a randomized, single-blinded fashion, receiving $0.15 \mathrm{IU}$ insulin $/ \mathrm{kg}$ body weight as an i.v. bolus. After hypoglycemia (serum glucose less than $2.2 \mathrm{mmol} / \mathrm{l}$ ) had been achieved, $500 \mathrm{ml}$ isotonic saline (protocol A (A)), or $500 \mathrm{ml} \mathrm{5 \%} \mathrm{glucose} \mathrm{solution} \mathrm{(protocol} \mathrm{B} \mathrm{(B))} \mathrm{were} \mathrm{infused} \mathrm{over} 30 \mathrm{~min}$. Results: Compared with saline, glucose infusion shortened the period of hypoglycemia from $31+14$ to $17+6 \mathrm{~min}(P<0.01)$. In addition, prolonged duration of hypoglycemia $(>45 \mathrm{~min})$ was reduced (6 subjects in protocol A vs none in protocol B). Despite shorter duration of hypoglycemia, all subjects had adequate stimulated cortisol $(>500 \mathrm{nmol} / \mathrm{l})$ and $\mathrm{hGH}(>5 \mu \mathrm{g} / \mathrm{l})$ levels. Mean peak concentrations of plasma ACTH (24 $12 \mathrm{pmol} / \mathrm{l}$ (A) vs $21 \pm 8 \mathrm{pmol} / \mathrm{l}$ (B)), serum cortisol $(690 \pm 83 \mathrm{nmol} / \mathrm{l}$ vs $634 \pm 83 \mathrm{nmol} / \mathrm{l})$ and serum hGH $(26 \pm 16 \mu \mathrm{g} / \mathrm{l}$ vs $22 \pm 13 \mu \mathrm{g} / \mathrm{l})$ were slightly, but not significantly lower. In contrast, glucose infusion significantly reduced peak plasma epinephrine levels at $45 \mathrm{~min}$ $(4.96 \pm 4.91 \mathrm{pmol} / \mathrm{l}(\mathrm{A})$ vs $1.53 \pm 1.1 \mathrm{pmol} / \mathrm{l}(\mathrm{B}), P<0.05)$ and ameliorated discomfort, as evaluated by a visual analog scale $(P<0.05)$.

Conclusions: Taken together, our pilot study suggests that, while the duration of hypoglycemia is shortened and acute epinephrine response is reduced, low-dose infusion of glucose does not significantly alter peak cortisol and growth hormone responses during ITT. Studies with a larger number of subjects and patients with suspected hypopituitarism are needed to further evaluate this modified protocol.
\end{abstract}

European Journal of Endocrinology 153 521-526

\section{Introduction}

Undiagnosed, and thus, untreated hypopituitarism is a potentially life threatening disease. The diagnosis is usually established by a combination of baseline endocrine parameters and various stimulation tests. The insulin tolerance test (ITT) is widely accepted as the gold standard for the evaluation of patients with suspected hypopituitarism (1-4). However, due to the necessity of close surveillance during the test, the procedure is highly time and effort consuming. To ensure safety, patients must be continuously monitored for their vital signs, vigilance and blood glucose values during at least $90 \mathrm{~min}$ (5). Furthermore, this test can be unpleasant for the patient and is potentially hazardous (4-8). Single fatal complications of the ITT have been described in children, including ventricular fibrillation in a child with a hereditary defect of the transmembraneous potassium transport and two cases of cerebral edema in children receiving large amounts of hyperosmolar glucose after critical hypoglycemia $(5-6)$.

We hypothesized that routine glucose infusion after critical hypoglycemia had been achieved, could markedly simplify the test, and improve safety and convenience for the patients. However, it is not clear whether 
the cortisol and growth hormone responses to hypoglycemic stress might be altered by shortening the hypoglycemic period. This implies that before routinely using such a modified test protocol, it has to be prospectively evaluated for safety, efficacy, and test reliability. Since demonstration of equivalence of the test parameters between the standard ITT protocol and a modified test with glucose infusion would require more than 50 subjects (corresponding to more than 100 ITTs), we performed a pilot study on healthy volunteers to gather preliminary evidence for test equivalence. As such, we performed a randomized, single-blinded pilot study on 16 healthy subjects investigating the effect of low-dose glucose infusion during ITT on time of hypoglycemia, neuroendocrine stress response and patient's discomfort.

\section{Subjects and methods}

\section{Subjects}

Sixteen healthy subjects (three females, and 13 males, age $25 \pm 5$ years, BMI $23.66 \pm 1.19 \mathrm{~kg} / \mathrm{m}^{2}$ ) participated in this study. Female volunteers were tested between the 7 th and the 14 th day of their menstrual cycle, while females on oral contraceptives were excluded from participation of the study. Written informed consent was obtained from every participant and the study was approved by the ethics committee of the University of Freiburg.

\section{Protocols}

All subjects underwent ITT on two occasions in a randomized, single-blinded fashion. The ITTs were started before $1000 \mathrm{~h}$ after an overnight fast for $10-14 \mathrm{~h}$. The subjects received a bolus i.v. insulin dose of $0.15 \mathrm{IU} / \mathrm{kg}$ body weight (ActrapidTM, NovoNordisk, Denmark). Blood samples were taken at $-15,0,30$, 45, 60 and $90 \mathrm{~min}$ after insulin administration for the determination of serum glucose, plasma ACTH, serum cortisol and serum growth hormone (hGH) levels. Plasma epinephrine, norepinephrine and dopamine concentrations were determined at 0,30 and $45 \mathrm{~min}$. Between 15 and $45 \mathrm{~min}$ after insulin administration, the serum glucose was determined every $5 \mathrm{~min}$, thereafter every $15 \mathrm{~min}$ with a photometric glucose analyzer (Beckman Coulter, Krefeld, Germany). A successful ITT was defined by a blood glucose nadir lower than $2.2 \mathrm{mmol} / \mathrm{l}$ and clinical symptoms of hypoglycemia, i.e. perspiration, fatigue, somnolence or hunger (7).

During protocol A, an infusion of $500 \mathrm{ml}$ isotonic $\mathrm{NaCl}$ solution over $30 \mathrm{~min}$ was started immediately after the serum glucose concentration had dropped to levels below $2.2 \mathrm{mmol} / \mathrm{l}$. During protocol B, subjects instead received $500 \mathrm{ml} \mathrm{5 \%}$ glucose solution over $30 \mathrm{~min}$. The interval between the two ITTs in an individual participant was not longer than 4 weeks. In 15 out of 16 subjects adequate hypoglycemia was achieved during ITT.
One male subject was excluded because critical hypoglycemia $<2.2 \mathrm{mmol} / \mathrm{l}$ and symptoms of hypoglycemia were not accomplished. The infusion of saline or glucose was generally well tolerated with the exception of one participant who described mild pain in the arm where he had received the glucose infusion. However, there were no signs of thrombophlebitis and the participant fully recovered within the same day without intervention.

\section{Assays}

Serum cortisol, serum hGH and plasma ACTH concentrations were determined by chemiluminescence using an autoanalyzer (Nichols AdvantageTM (Nichols Institute, San Juan Capistrano, CA, USA), with intraassay variations and inter-assay variations of $1.2-$ $4.2 \%$ and $6.4-8.4 \%$ respectively). Accuracy of the assay procedure over the study period was documented by daily calibration and by regular determination of reference plasma samples. A normal adrenal response to ITT was defined as a peak serum cortisol concentration $\geq 500 \mathrm{nmol} / \mathrm{l}(1,6,9)$, and a normal hGH secretion was defined as peak hGH levels exceeding $5 \mu \mathrm{g} / \mathrm{l}(10)$. Plasma catecholamines were determined in a subset of 11 subjects by high pressure liquid chromatography followed by fluorescence detection (PerkinElmer, Wellesley, MA, USA).

\section{Questionnaires}

To quantify the extent of the symptoms and complaints associated with the ITTs we used a questionnaire which covered common symptoms and complaints usually experienced during hypoglycemia including nausea, sweating, trembling, hunger, anxiety, vertigo, lack of concentration, visual problems, fatigue and nausea. The questionnaire had to be completed within $24 \mathrm{~h}$ after the ITT. Visual analogue scales with $100 \mathrm{~mm}$ bars were used to quantify the complaints. A mark at $0 \mathrm{~mm}$ indicated no complaints, whereas a mark at $100 \mathrm{~mm}$ indicated the most severe complaints.

\section{Statistics}

All values represent means \pm S.D, if not stated otherwise. The area under the curve (AUC) for time concentration was calculated for glucose, ACTH, cortisol, and growth hormone from -15 to $90 \mathrm{~min},-15$ to $30 \mathrm{~min}$ and 30 to $90 \mathrm{~min}$. In addition, a global symptom score was calculated summing up the ten items of the questionnaire. Statistical significance of the differences between protocols A and B was calculated using paired $t$-test and ANOVA after logarithmic transformation, as appropriate. Correlation between time of hypoglycemia and the stress hormone response was assessed by Spearman's rank test. The statistical analyses were calculated using the BMDP statistical software package. 


\section{Results}

As expected, the nadir serum glucose concentration $(1.6 \pm 0.4 \mathrm{mmol} / \mathrm{l}$ on protocol A and $1.6 \pm 0.2 \mathrm{mmol} / \mathrm{l}$ on protocol $\mathrm{B} ; \mathrm{P}=0.25)$ and the time until hypoglycemia $<2.2 \mathrm{mmol} / \mathrm{l}$ was reached $(20 \pm 5 \mathrm{~min}$ in protocol $\mathrm{A}$ and $20 \pm 3 \mathrm{~min}$ in protocol $\mathrm{B}, P=0.9$ ) were equivalent on both protocol. However, the duration of hypoglycemia below $2.2 \mathrm{mmol} / \mathrm{l}$ was significantly reduced by glucose administration $(31 \pm 14 \mathrm{~min}$ in protocol A (range 10-60 min) and $17 \pm 6 \mathrm{~min}$ in protocol B (range 10-30 min); $P<0.01$, Fig. 1A). Accordingly, prolonged duration of hypoglycemia (45 min and more) occurred

A

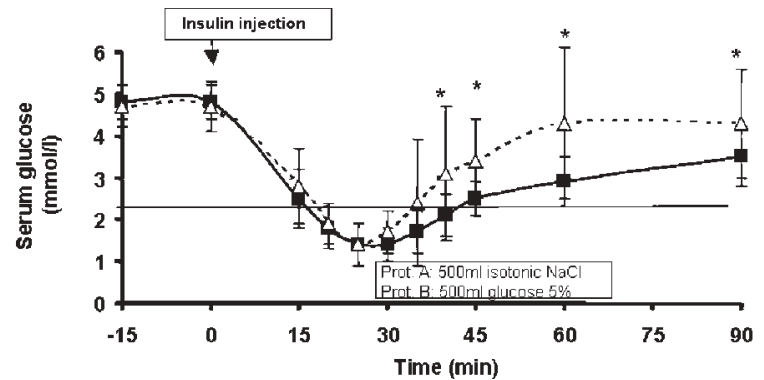

B

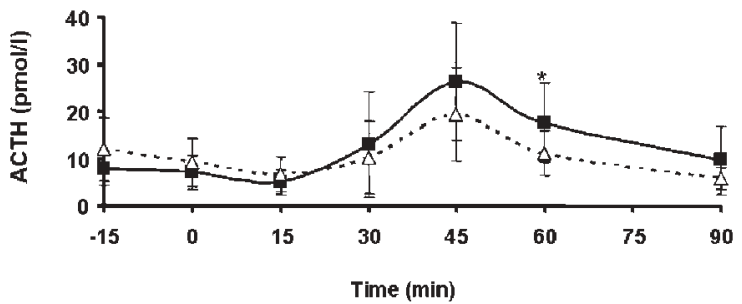

C

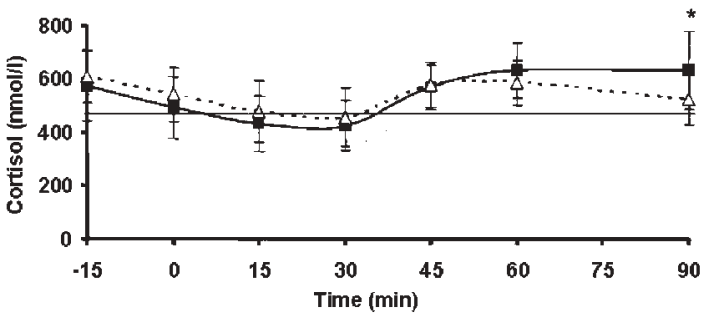

D

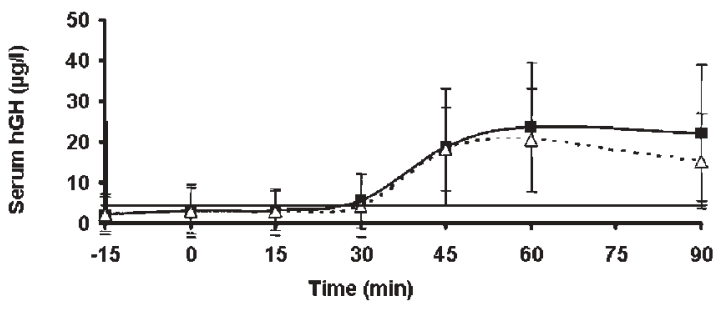

Figure 1 Mean ( \pm S.D.) serum glucose (A), plasma ACTH (B), serum cortisol (C) and serum hGH (D) concentrations during protocol A $(\square)$ and protocol B $(\triangle)$. Glucose nadir was reached after $20 \pm 5 \mathrm{~min}$ in protocol $A$ and after $20 \pm 3 \mathrm{~min}$ in protocol B. Horizontal line represents the thresholds for hypoglycemia (A), cortisol (C) and growth hormone (D) concentrations respectively. in six subjects during protocol A but in none during protocol B. As expected, the AUC from -15 to 90 min of protocol A was significantly lower than that of protocol B $(317+212$ vs $384+454 \mathrm{mmol} / \mathrm{l} / \mathrm{min}$ respectively, $P=0.002$ ) which was entirely due to highly significant differences between the AUC from 30 to $90 \mathrm{~min}$ $(P=0.0007)$.

All 15 subjects had adequate cortisol $(>500 \mathrm{nmol} / \mathrm{l})$ responses upon hypoglycemic stress in both protocols (peak serum cortisol concentration: $690 \pm 83 \mathrm{nmol} / \mathrm{l}$ (range 513-784 nmol/l) in protocol A vs $634 \pm 83 \mathrm{nmol} / \mathrm{l}(508-726 \mathrm{nmol} / \mathrm{l})$ in protocol B; $P$, not significant, Fig. 1C and 2). Unexpectedly, one subject reached a peak hGH concentration of only $2 \mu \mathrm{g} / \mathrm{l}$ during conventional ITT but showed a sufficient hGH response of $6 \mu \mathrm{g} / \mathrm{l}$ in the modified protocol B. All other subjects, however, had adequate hGH levels without significant differences between both protocols (peak serum hGH concentration: $26 \pm 16 \mu \mathrm{g} / \mathrm{l}$ (range 2-50) in protocol A vs $22 \pm 13 \mu \mathrm{g} / \mathrm{l}(6-37.5 \mu \mathrm{g} / \mathrm{l})$ in protocol B; $P$, not significant, Fig. 2). After reaching the peak values, ACTH, serum cortisol, and hGH levels tended to be lower at

A

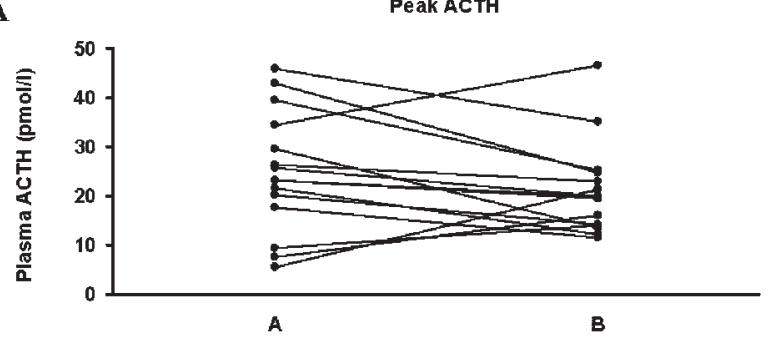

B

Peak Cortisol
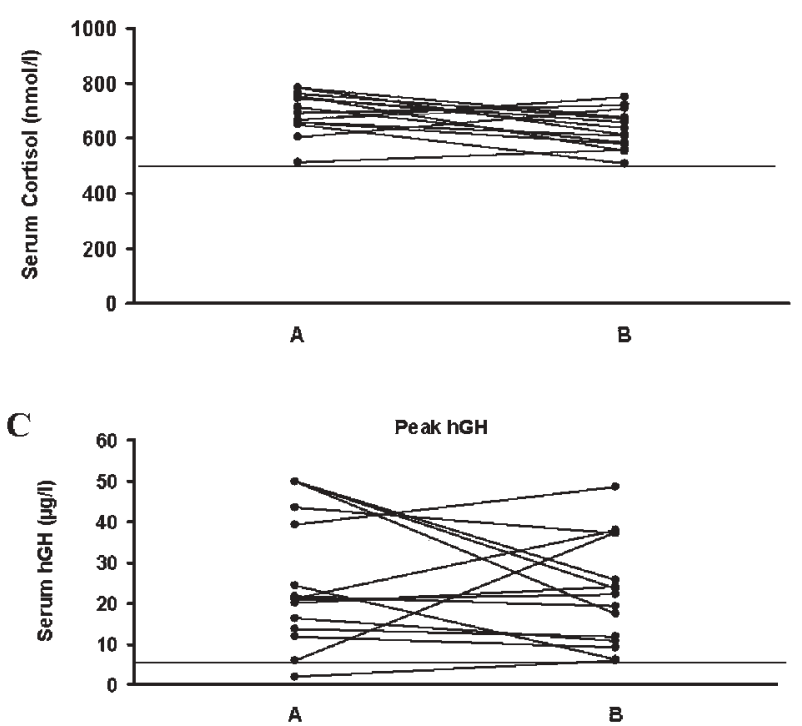

Figure 2 Individual peak plasma ACTH (A), serum cortisol (B) and serum hGH $(C)$ concentrations in 15 subjects during protocol A (saline i.v.) and protocol B (glucose i.v.) during the insulin tolerance test. Horizontal line represents the threshold for secondary adrenal insufficiency (B) and hGH deficiency (C). 

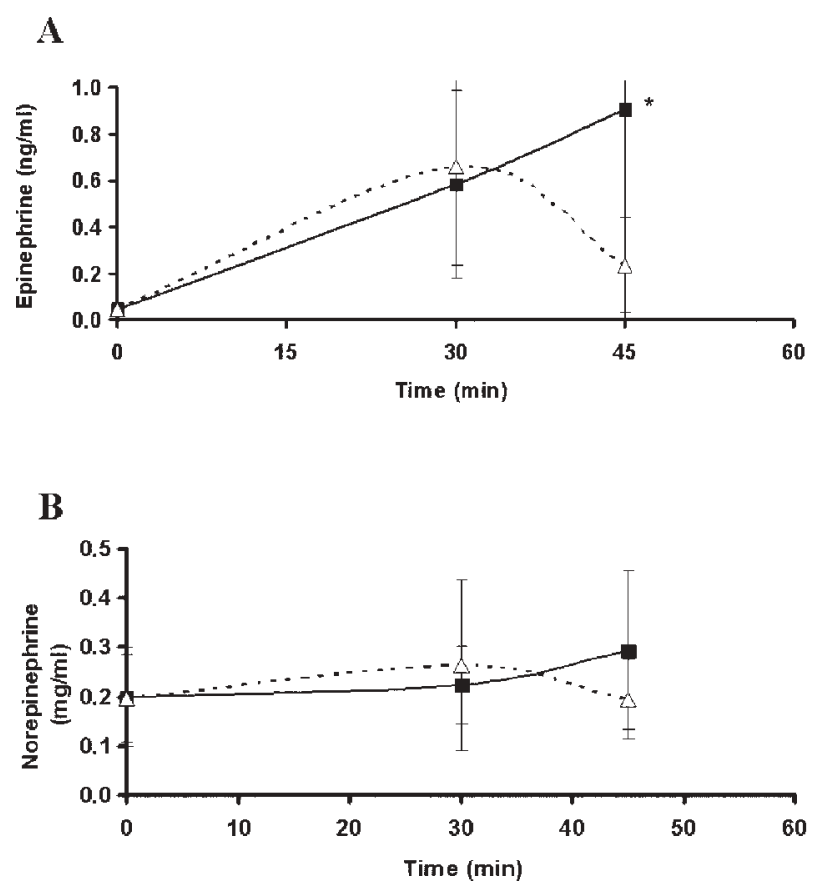

Figure 3 Plasma epinephrine $(A)$ and norepinephrine $(B)$ (means \pm S.D.) during protocol $\mathrm{A}(\boldsymbol{\square})$ and protocol $\mathrm{B}(\triangle)(n=11$, one female and ten male subjects).

$60 \mathrm{~min}$ and $90 \mathrm{~min}$ during protocol B. However, the AUC for ACTH, cortisol and hGH did not differ significantly between protocols A and B. Although glucose levels were different between the two protocols, there was no significant correlation between the duration of hypoglycemia and the maximum ACTH, cortisol and hGH concentration (maximum ACTH: $r=0.26, P=0.19$; maximum cortisol: $r=0.34, P=0.78$; maximum hGH: $r=0.21$, $P=0.29$ ).
During the first $30 \mathrm{~min}$ of the test, plasma catecholamine concentrations were comparable between both protocols (at $30 \mathrm{~min}$ : epinephrine $3.18 \pm 2.2 \mathrm{pmol} / \mathrm{l}$ vs $3.62 \pm 2.34 \mathrm{pmol} / \mathrm{l} ; P$, not significant; norepinephrine $1.32 \pm 0.46 \mathrm{pmol} / \mathrm{l}$ vs $1.56 \pm 1.03 \mathrm{pmol} / \mathrm{l}$; $P$, not significant; dopamine $0.4 \pm 0.22 \mathrm{pmol} / \mathrm{l}$ vs $0.41 \pm 0.31 \mathrm{pmol} / \mathrm{l}$; $P$, not significant, Fig. 3A-B). However, plasma epinephrine levels continued to rise after $30 \mathrm{~min}$ during protocol A, but significantly declined during protocol B (45 min: epinephrine $4.96 \pm 4.91 \mathrm{pmol} / \mathrm{l}$ vs $1.53 \pm 1.1 \mathrm{pmol} /$; $P<0.05)$. Norepinephrine and dopamine concentrations did not show significant differences.

The questionnaire for assessment of symptoms of hypoglycemia indicated that the main complaints during ITT were fatigue, hunger, sweating and lack of concentration. Although no statistically significant difference could be observed for each symptom separately, the mean total score was significantly lower in protocol B $(10.3+13.4$ vs $13.8+13.3 \mathrm{~mm}$ in $\mathrm{A}$, $P=0.04$, Fig. 4).

\section{Discussion}

Glucose intervention has been introduced as an established part of the ITT protocol at some centers. Lange et al. (4) applied glucose only in patients with prolonged hypoglycemia, whereas Hoeck et al. (11) administered 50 g glucose orally to every patient after reaching critical hypoglycemia. The 'Barts endocrine protocols' recommend the application of glucose in patients with severe or prolonged hypoglycemia or with complications from hypoglycemia (12). Donaubauer et al. (13) investigated, in a small study, repeated ITTs in three healthy subjects (age 17-24 years, 0.1 IU insulin/kg body weight i.v.) with and without interruption of hypoglycemia by an infusion of $100 \mathrm{ml}$ glucose

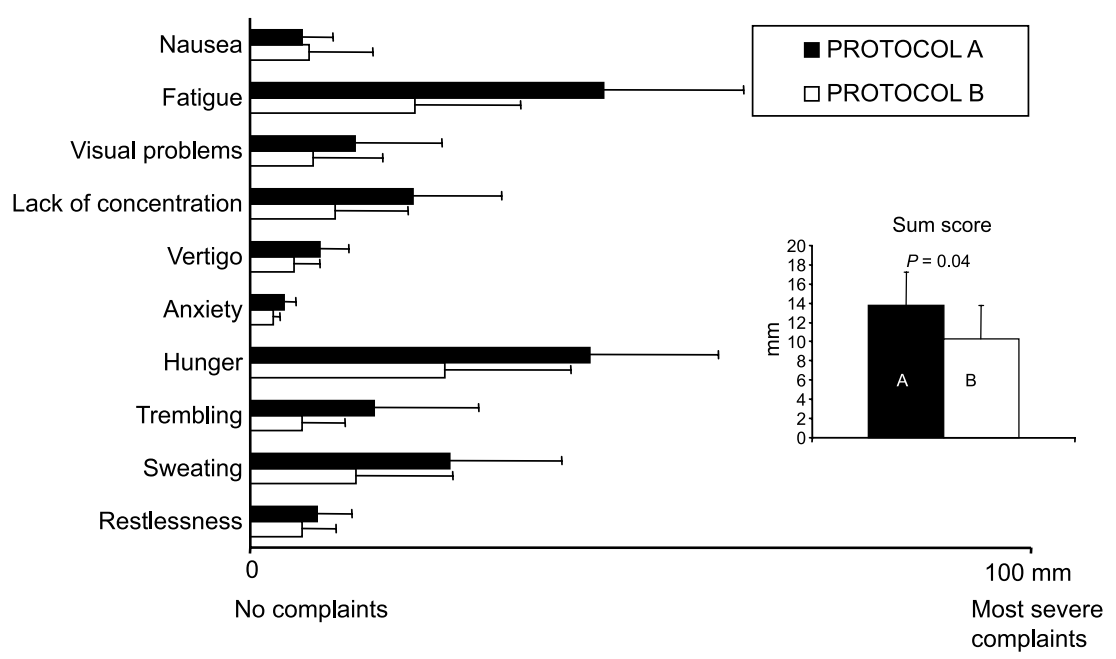

Figure 4 Mean symptoms (+S.D.) and mean total symptom score (+S.E.M.) associated with the insulin tolerance test assessed by questionnaire. To quantify the complaints, visual analogue scales with $100 \mathrm{~mm}$ bars were used, with $0 \mathrm{~mm}$ expressing no complaints, and $100 \mathrm{~mm}$ expressing the most severe complaints. 
$20 \%$ solution. However, to our knowledge, there has been no formal investigation whether glucose intervention can be routinely done without affecting the responses of cortisol or growth hormone.

To investigate the influence of low-dose glucose infusion during the ITT on the duration of hypoglycemia, on the neuroendocrine and adrenomedullary stress response and on discomfort experienced by the subjects on a broader scale, we performed a randomized, single-blinded study in 16 healthy volunteers who underwent ITT according to two different protocols. Whereas in protocol A subjects received isotonic saline infusion, they received $5 \%$ glucose infusion over $30 \mathrm{~min}$ in protocol $\mathrm{B}$.

One of the disadvantages of the conventional ITT is the need for long surveillance of the patients during and after reaching the glycemic nadir. Remarkable in this regard is the extended time of hypoglycemia in some of the healthy subjects for up to $60 \mathrm{~min}$ during protocol A. Early glucose infusion significantly shortened the time of hypoglycemia from a mean of 31 to $17 \mathrm{~min}$ and especially reduced prolonged duration of hypoglycemia, thus increasing safety of the applied test.

The main concern against ameliorating the effects of hypoglycemia through glucose administration is that it might adversely affect test parameters of the ITT. For example, in the study by Donaubauer et al. (13) one subject would have been misdiagnosed to have secondary adrenal insufficiency and growth hormone deficiency using early glucose infusion. The authors concluded that early interruption of hypoglycemia might increase the number of false positive results. However, in this study subjects did not experience hypoglycemic symptoms applying the protocol with early glucose infusion which indicates that nadir glucose concentrations might have been affected in this protocol. In our study, all participants had symptoms of hypoglycemia. In addition, nadir glucose concentrations were not different between groups. Accordingly, although mean cortisol and hGH concentrations were lower at the end of the test $(60$ and $90 \mathrm{~min}$ ) when applying glucose infusion, peak cortisol and hGH concentrations and the AUC of these hormones were not significantly different between the protocols. Furthermore, no subject was misclassified to have secondary adrenal insufficiency or hGH deficiency in our study.

Landon and colleagues (14-17) published several studies investigating the insulin tolerance test in healthy subjects and in patients with hypopituitarism, pituitary tumors, acromegaly and anorexia nervosa. They described a significant correlation with the value of glucose nadir and the response of cortisol and growth hormone. Cryer et al. (18) proved in several studies investigating diabetic patients that the absolute value of glucose nadir determines the extent of counterregulatory responses. The studies of Landon et al. and Cryer et al. support our hypothesis, that the duration of hypoglycemia in the insulin tolerance test can be shortened by early glucose intervention without affecting the response of cortisol and growth hormone if the glucose nadir is maintained.

As expected, the serum epinephrine concentrations markedly increased during hypoglycemia. The beneficial effect of glucose infusion was evident during protocol B when plasma epinephrine concentrations declined after $30 \mathrm{~min}$ whereas they remained high in protocol A. Similar, but less pronounced changes were found for norepinephrine and dopamine levels. One of the known complications of ITT in older subjects is an impaired coronary blood flow and increased myocardial oxygen consumption as a result of the adrenomedullary stress response. This may induce acute coronary syndrome or even myocardial infarction, if the test is performed in patients with unrecognized coronary heart disease. Although we tested only a few subjects, our data strongly indicate that in this respect protocol $\mathrm{B}$ is less stressful than protocol A and might be especially useful for older subjects.

The visual analogue scales used to quantify the extent of hypoglycemia demonstrated that fatigue, hunger, sweating, and disability to concentrate were the main symptoms during both protocols. Overall, all subjects described less complaints in nine out of ten evaluated parameters during the modified protocol $\mathrm{B}$, and the total symptom score was significantly lower. This is most likely due to a shorter duration of neurohypoglycemia, although lower plasma catecholamine levels may have contributed to a reduced level of discomfort. In patients with hypopituitarism, a longer duration of hypoglycemia is likely to occur because of a reduced contrainsulinary stress hormone response. Thus, it can be expected that those patients might experience more complaints during and after prolonged hypoglycemia than normal subjects. Especially in older patients with pre-existing atherosclerosis, prolonged duration of hypoglycemia exposes these subjects to a higher risk of unforeseen complications such as seizure, stroke and acute coronary syndrome.

Overall, our data suggest that ITT with standardized glucose infusion after critical hypoglycemia might reduce discomfort and possibly other complications, whereas diagnostic accuracy seems to be sustained. As stated, our study was intended as a pilot study to obtain preliminary evidence of equivalence for the two protocols tested. In this respect, the results of our study are promising. A much larger study is necessary to definitely rule out inferior test characteristics of the modified test. As long as these data have not been generated, we suggest to restrict glucose infusion to those patients, who might have an increased risk for hypoglycemic complications. In addition, our protocol needs to be evaluated in patients with hypopituitarism comparing it to standard pituitary function tests, such as ACTH stimulation test and GHRH/arginine stimulation 
test. As uncorrected growth hormone deficiency and (secondary) adrenal insufficiency can result in low glucose levels, thus, indirectly increasing insulin sensitivity, in such a setting, a lower dose of insulin $(0.05-0.1 \mathrm{U} / \mathrm{kg})$ should be used. This might lead to the definition of adjusted cut-off values for cortisol or growth hormone responses suitable for our modified ITT protocol.

\section{Funding}

This study was supported by a research grant awarded by the German Endocrine Society to F B.

\section{References}

1 Arlt W \& Allolio B. Adrenal Insufficiency. Lancet 2003361 1881-1892.

2 Tuchelt H, Dekker V, Bähr V \& Oelkers W. Dose-response relationships between plasma ACTH and serum cortisol in the insulin hypoglycemia test in 25 healthy subjects and 109 patients with pituitary disease. Clinical Endocrinology 200053 301-307.

3 Erturk E, Jaffe CA \& Barkan AL. Evaluation of the integrity of the hypothalamic-pituitary-adrenal axis by insulin hypoglycemia test. Journal of Clinical Endocrinology and Metabolism $1998 \mathbf{8 3}$ $2350-2354$

4 Lange M, Svendsen OL, Skakkebaek NE, Mülller J, Juul A, Schmiegelow A \& Feldt-Rasmussen U. An audit of the insulin-tolerance test in 255 patients with pituitary disease. European Journal of Endocrinology 2002147 41-47.

5 Grinspoon SK \& Biller BMK. 1994 Laboratory assessment of adrenal insufficiency. Journal of Clinical Endocrinology and Metabolism 200279 923-931.

6 Oelkers W. Adrenal Insufficiency (Review). New England Journal of Medicine 1996335 1206-1212.

7 Binder G, Martin D, Bosk A \& Ranke MB. Lebensbedrohliche Hypokaliämie mit hämodynamischem Herzstillstand bei einem Wachstumshormonstimulationstest mit Insulin. Abstract. Monatsschrift Kinderheilkunde 2002 V11.

8 Shah A, Stanhope R \& Matthew D. Hazards of pharmacological tests of growth hormone secretion in childhood. British Medical Journal 1992304 173-174.
9 Dullaart RP, Pasterkamp SH, Beentjes JA \& Sluiter WJ. Evaluation of adrenal function in patients with hypothalamic and pituitary disorders: comparison of serum cortisol, urinary free cortisol and the human-corticotrophin releasing hormone test with the insulin tolerance test. Clinical Endocrinology 199950 465-471.

10 Growth hormone research society. Consensus guidelines for the diagnosis and treatment of adults with growth hormone deficiency: summary statement of the growth hormone research society workshop on adult growth hormone deficiency. Journal of Clinical Endocrinology and Metabolism 199883 379-381.

11 Hoeck HC, Vestergaard P \& Jakobsen PO. Diagnosis of growth hormone deficiency in adults with hypothalamic-pituitary disorders: comparison of test results using pyridostigmine plus GH-releasing hormone (GHRH), clonidine plus GHRH, and insulin induced hypoglycemia as GH secretagogues. Journal of Clinical Endocrinology and Metabolism 200085 1467-1472.

12 Trainer PJ \& Besser GM. The Barts Endocrine Protocols, pp 84-87. Edinburgh: Churchill Livingstone, 1995.

13 Donaubauer J, Kratzsch J, Willgerodt H, Kiess W, Nowak T \& Keller E. Importance of hypoglycemic symptoms in insulin tolerance test. Growth Hormone and IGF Research $2000 \mathbf{1 0}$ $172-182$.

14 Greenwood FC, Landon J \& Stamp TCB. The plasma sugar, free fatty acid, cortisol and growth hormone response to insulin. I. In control subjects. Journal of Clinical Investigations $1966 \mathbf{4 5}$ $429-436$.

15 Landon J, Greenwood FC, Stamp TCB \& Wynn V. The plasma sugar, free fatty acid, cortisol and growth hormone response to insulin, and the comparison of this procedure with other tests of pituitary and adrenal function. II. In patients with hypothalamic or pituitary dysfunction or anorexia nervosa. Journal of Clinical Investigations $1966 \mathbf{4 5} 437-449$.

16 Greenwood FC \& Landon J. Assessment of hypothalamic pituitary function in endocrine disease. Journal of Clinical Pathology 1966 $19284-292$.

17 Landon J, Wynn V \& James VH. The adrenocortical response to insulin induced hypoglycemia. Journal of Endocrinology 196327 $183-192$.

18 Cryer P. Hierarchy of physiological responses to hypoglycemia: relevance to clinical hypoglycemia in type 1 diabetes mellitus. Hormone and Metabolic Research 199729 92-96.

Received 7 April 2005

Accepted 27 June 2005 